\section{Ultrasound biomicroscopy in deep sclerectomy}

HA Khairy ${ }^{1,2}$, HR Atta1 , FD Green ${ }^{1}$, J van der Hoek ${ }^{1}$ and A Azuara-Blanco'

Keywords: ultrasound biomicroscopy; deep sclerectomy; nonpenetrating trabeculectomy; glaucoma

Purpose To evaluate by ultrasound characteristics and the intraocular pressure (IOP) lowering mechanisms of deep sclerectomy after long-term follow-up. Methods In all, 22 eyes of 21 consecutive patients who had deep sclerectomy were examined by UBM. Several UBM variables were prospectively evaluated, including the presence and maximum length and height of the intrascleral space, the minimum thickness of residual trabeculo-Descemet membrane (TDM), the presence and type of subconjunctival filtering bleb, and the presence of other possible drainage sites, for example suprachoroidal. Surgical success was considered to be achieved when the IOP was $<22 \mathrm{mmHg}$ and the IOP was lowered by more than $20 \%$ without the use of any medication. The possible association between UBM variables and the surgical outcome was determined.

Results The mean time between surgery and the UBM examination was $12.0 \pm 5.3$ months. The mean IOP decreased significantly from a preoperative value of $23.7 \pm 4.0$ to $16.0 \pm 3.9 \mathrm{mmHg}$ at the time of UBM $(P<0.01)$. There was a poor correlation between the level of IOP at the time of UBM and the length of intrascleral space $\left(r^{2}=0.0016\right)$, the height of the intrascleral space $\left(r^{2}=0.136\right)$, or the thickness of remaining TDM $\left(r^{2}=0.0009\right)$. The presence and type of filtering bleb were not associated with the success.

Conclusions In patients undergoing deep sclerectomy, UBM examination after long-term follow-up showed the presence of an intrascleral space and a filtering bleb in most patients. The surgical outcome was not associated with the UBM variables of the surgical area.

Eye (2005) 19, 555-560. doi:10.1038/sj.eye.6701558

Published online 11 March 2005

\section{Introduction}

The main advantages of nonpenetrating trabeculectomy (NPT) over conventional trabeculectomy rely on the fact that NPT avoids full-thickness penetration into the anterior chamber. In theory, this would markedly reduce the risk of postoperative complications due to overfiltration and hypotony. It is possible that NPT may also result in less cataract progression, which would be a very significant advantage, particularly in the context of filtrating surgery in developing countries. ${ }^{1}$

Several techniques of NPT have been described. In 1962, Kranzov ${ }^{2}$ performed the first sinusotomy by removing a lamellar band of the sclera, opening Schlemm's canal leaving in place the trabeculum and the inner wall of the canal. Sinusotomy never became popular because it was a difficult operation and the surgical results were not convincing. ${ }^{3}$ Zimmemann et al ${ }^{4}$ in 1984 described Ab-externo Trabeculectomy in which a superficial scleral

flap was created and the inner wall of Schlemm's canal and the juxtacanalicular trabeculum (where the major resistance of aqueous outflow was thought to exist) were removed.

Fyodorov ${ }^{5}$ and Kozlov ${ }^{6}$ in the late 1980's first used the term 'deep sclerectomy'. Several articles on deep sclerectomy describe several surgical variants. ${ }^{3,7-9}$ The use of scleral implants and antimetabolites has been proposed to improve the surgical outcome. ${ }^{3,7}$ Additionally, postoperative goniopuncture performed with a neodymium:YAG (Nd:YAG) laser may further contribute to an improved outflow by transforming a nonpenetrating operation into a penetrating one. ${ }^{9}$

A further development of NPT, et $a l^{10}$ in 1999. In viscocanalostomy, the cut viscocanalostomy, was introduced by Stegmann
${ }^{1}$ Department of Ophthalmology, Aberdeen Royal Infirmary, University of Aberdeen, Aberdeen, UK

${ }^{2}$ Ophthalmology Department, Menoufyia University, Egypt

Correspondence: HA Khairy, Department of Ophthalmology, Aberdeen Royal Infirmary, University of Aberdeen, Aberdeen AB25 2ZN, UK Tel: +44 1224 553217; Fax: +44 1224849156 E-mail: khairyhany@ hotmail.com

Received: 19 November 2003

Accepted: 26 February 2004 Published online: 11 March 2005 
ends of the canal are then expanded with a

viscoelastic material. Viscoelastic is also injected into the region of excised sclera, or scleral lake, to prevent healing. ${ }^{10}$

Both deep sclerectomy and viscocanalostomy involve fashioning of a partial thickness scleral flap, removing a second layer of sclera deep to the initial flap, and exposing Descemet's membrane. Schlemm's canal is unroofed during removal of the second, deep scleral layer.

Ultrasound biomicroscopy (UBM) provides details of the anterior chamber, ciliary body, and corneoscleral limbal tissues not obtained by other methods in the living eyes. ${ }^{11}$ Previous studies suggested that UBM could provide a more useful understanding of the mechanism of IOP reduction after glaucoma surgery. ${ }^{11-15}$ To the best of our knowledge, there are no published UBM investigations on aqueous pathways after deep sclerectomy without scleral implant.

\section{Methods}

In all, 22 eyes of 21 consecutive patients, all were white Caucasian (11 male and 10 female patients) who had deep sclerectomy, were included in this study. Subjects were enrolled in this study after giving written and informed consent to participate, and the Tenets of the Declaration of Helsinki were adhered to. The IOP lowering mechanisms were investigated by exploring the surgical area with UBM. Patients included in this study had uncontrolled glaucoma despite maximal medical therapy and they had no history of laser treatment or previous ocular surgical intervention. In all, 17 patients had primary open angle glaucoma, two had pseudoexfoliative glaucoma, and two had low-tension glaucoma.

Informed consent was obtained, and full ophthalmic examination was performed the day before surgery and, postoperatively, at $1,3,6,12$, and 18 months. The assessment included visual acuity, IOP measurement, slit-lamp examination, and dilated fundus biomicroscopy.

The surgery was performed in all patients by the same surgeon (FDG). A fornix-based conjunctival flap was prepared. A $5 \times 5$ scleral flap including one-third of scleral thickness, was dissected $1-1.5 \mathrm{~mm}$ into clear cornea. A second $4 \times 4$ deeper scleral flap was resected to allow deroofing of the Schlemm's canal and expose the Descemet's membrane. The remaining scleral layer was left as thin as possible. Afterwards, the superficial flap was closed tightly with 10-0 nylon sutures. Surgery was performed without the use of antimetabolites, scleral implants, or viscoelastic.
'Surgical success' was defined as an IOP $<22 \mathrm{mmHg}$ and a reduction in IOP of more than $20 \%$ without the use of any medication. 'Qualified success' was defined as an IOP $<22 \mathrm{mmHg}$ and an IOP reduction of more than $20 \%$ with the use of one or two topical medications. The IOP considered to define 'success' was that obtained nearest to the time of the UBM examination.

A Zeiss Humphrey UBM 840 (Paradigm Medical Industries, INC) system was used to provide highfrequency $(50 \mathrm{MHz})$ ultrasonic scan images. Scans were performed by an experienced investigator (HRA), using the technique developed by Pavlin et al. ${ }^{11}$ With the patient in the supine position and with the aid of an emersion scleral shell and an examination gel, the surgical area was scanned with the UBM probe. Radial and transverse sections of the sclerectomy area at 12 $\mathrm{o}^{\prime}$ clock were explored, using optimal $\mathrm{dB}$ gain that allowed best resolution and quality of images. Biometric measurements of the surgical site were performed with the electronic callipers installed in the instrument. The 'Segment calliper' resolution is $+5 \mu \mathrm{m}$. From longitudinal and transverse scans, the following variables were assessed:

- The presence and maximum length and height of the intrascleral lake.

- The minimum thickness of residual trabeculo-Descemet membrane (TDM).

- The presence and type of subconjunctival filtering bleb.

- The possibility of suprachoroidal drainage as an alternative aqueous pathway.

The measurements and assessment of other UBM variables were carried out independently by two observers (HRA and HAK). Both of them were masked to the IOP and the surgical outcome. Filtering blebs, when found, were classified into four categories according to the classification of Yamamoto et al ${ }^{14}$ : L-type (low reflective), H-type (high reflective), E-type (encapsulated), and F-type (flattened) (Figures 1-4).

The reflectivity of the tissues around the sclerectomy area was judged subjectively as hyporeflective or hyperreflective compared to the normal sclera of the same eye and on the same image. The presence of suprachoroidal or supraciliary hyporeflective was considered an evidence of possible suprachoroidal drainage.

Quantitative variables were compared using the Student's t-test. Linear regression analysis was used to analyse the relationship between the IOP, length and height of the intrascleral space, and thickness of TDM. $\chi^{2}$ was used to analyse the relationship between surgical success and the type of filtering bleb. 


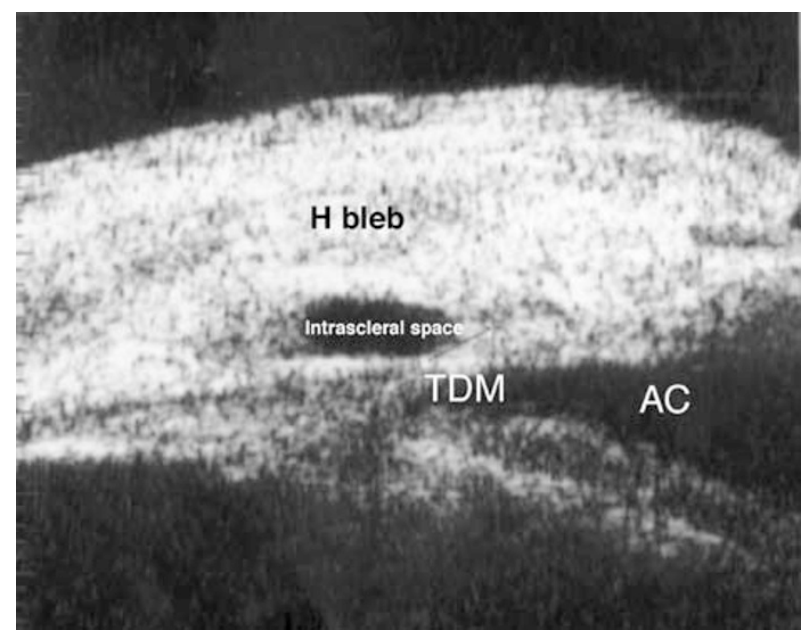

Figure 1 Longitudinal UBM examination of the surgical area after deep sclerectomy. A filtering bleb of H-type (high reflective) and an intrascleral space are observed. $A C=$ anterior anterior chamber and TDM $=$ trabeculo-descemet membrane.

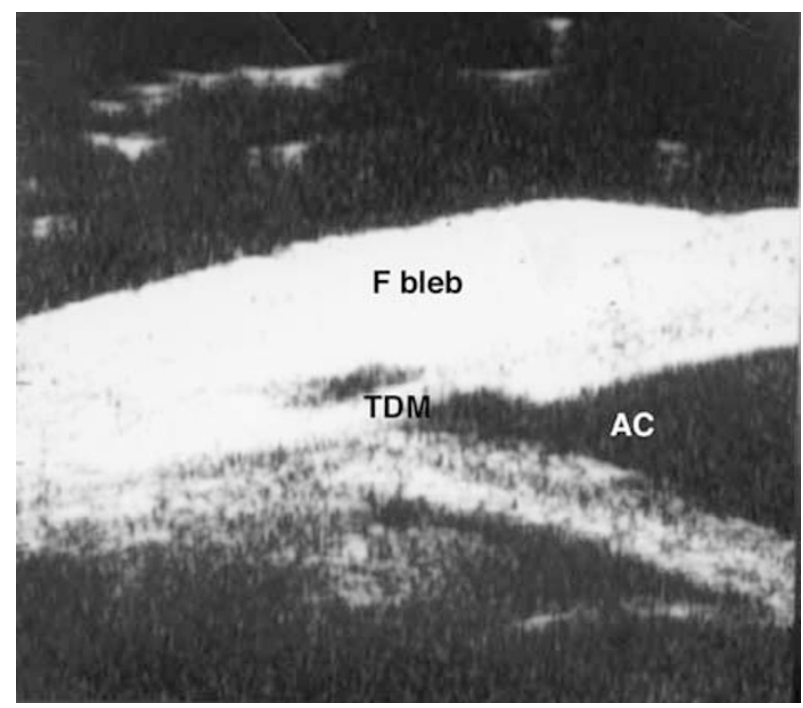

Figure 2 Longitudinal UBM examination of the surgical area after deep sclerectomy. A filtering bleb of F-type (flat) and an intrascleral space are observed. $\mathrm{AC}=$ anterior chamber and $\mathrm{TDM}=$ trabeculo-descemet membrane.

\section{Results}

All patients were white Caucasians. The mean time between surgery and the UBM examination was $12.0 \pm 5.3$ months (range 5-26 months). The mean time between UBM examination and tonometry was $6.9 \pm 4.0$ days. The mean IOP decreased significantly from a preoperative value of $23.7 \pm 4.0 \mathrm{mmHg}$ to a postoperative value of $16.0 \pm 3.9 \mathrm{mmHg}$ (range 11$26 \mathrm{mmHg})$ at the time of UBM $(P<0.01)$. The mean number of medications used before surgery was $2.2 \pm 1.0$ and the mean number of medications at the last followup after surgery was $1.4 \pm 0.7(P<0.05)$. Complete

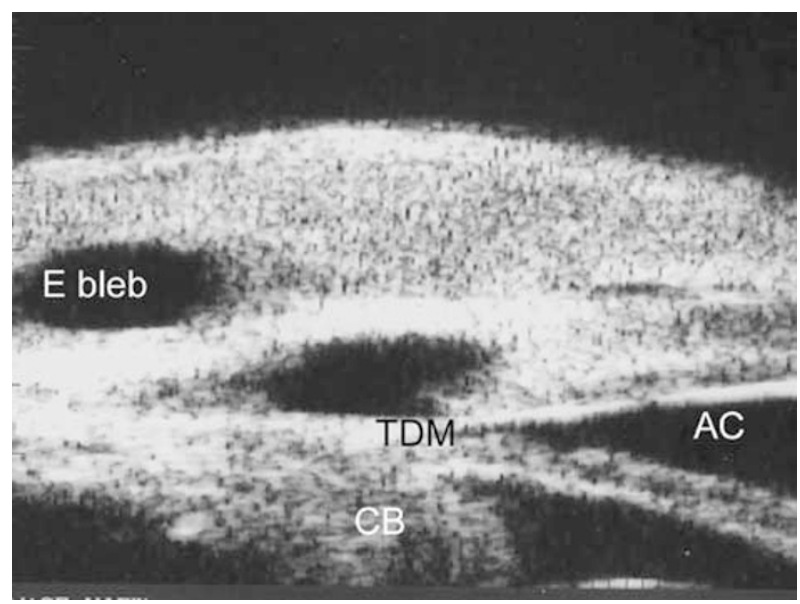

Figure 3 Longitudinal UBM examination of the surgical area after deep sclerectomy. A filtering bleb of E-type (encapsulated) and an intrascleral space are observed. $A C=$ anterior chamber, $\mathrm{TDM}=$ trabeculo-descemet membrane and $\mathrm{CB}=$ ciliary body.

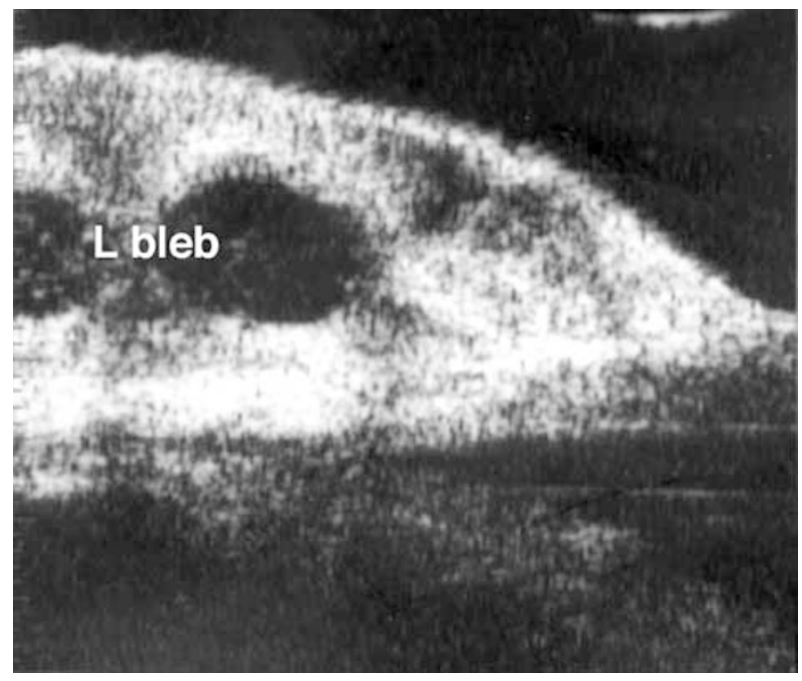

Figure 4 Longitudinal UBM examination of the surgical area after deep sclerectomy. A filtering bleb of L-type (low reflective) and an intrascleral space are observed.

surgical success (at the time of UBM examination) was achieved in 13 eyes (59.1\%). Qualified success was achieved in another six eyes $(27.3 \%)$. Microperforation of TDM occurred in one case and ciliary body prolapse in another, but it did not prevent completion of the operation. There were no cases of hyphaema, anterior chamber shallowing, choroidal detachment, or other late complications with the exception of failure of the operation.

Regarding UBM findings, in 20 eyes a wellcircumscribed, intrascleral space of variable reflectivity was present (Table 1). A thin hyper-reflective straight line extending from the peripheral cornea to the scleral spur separated the anterior chamber and the intrascleral space 
Table 1 Frequencies of surgical success, presence of intrascleral space and filtering blebs after deep sclerectomy

\begin{tabular}{lrc}
\hline Parameter & Number of eyes & Percentage (\%) \\
\hline Surgical success & & \\
Complete success & $13 / 22$ & 59.1 \\
Qualified Success & $6 / 22$ & 27.3 \\
Failure & $3 / 22$ & 13.6 \\
UBM variables present & & \\
Intrascleral space & $20 / 22$ & 90.9 \\
Filtering bleb & $20 / 22$ & 90.9 \\
H-type bleb & $11 / 22$ & 50.0 \\
F-type bleb & $6 / 22$ & 27.3 \\
L-type bleb & $2 / 22$ & 9.1 \\
E-type bleb & $1 / 22$ & 4.5 \\
\hline
\end{tabular}

Table 2 UBM measurements of the thickness of the TDM and the length and height of the intrascleral lake

\begin{tabular}{lccc}
\hline Measurements & $\begin{array}{c}\text { Obsever 1 } \\
\text { mean } \pm S D\end{array}$ & $\begin{array}{c}\text { Obsever 2 } \\
\text { mean } \pm S D\end{array}$ & P-value \\
\hline TDM thickness & $0.26 \pm 0.09$ & $0.25 \pm 0.09$ & 0.406 \\
Length of the space & $1.62 \pm 0.69$ & $1.63 \pm 0.71$ & 0.459 \\
Height of the space & $0.43 \pm 0.14$ & $0.42 \pm 0.14$ & 0.301 \\
\hline
\end{tabular}

(ie, TDM). A subconjunctival filtering bleb of different reflectivity was detected by UBM in these same patients (Table 1).

All four types of blebs were detected, the H-type (high reflective) being the most common. Surgery failed in three patients; two of them had an H-type bleb and another had no bleb. One successful case had no filtering bleb. There was no association between the surgical success as defined above and the type of filtering bleb $(P>0.05)$. Neither suprachoroidal hyporeflective area nor TDM perforation was detected in any of the examined eyes.

There was a $100 \%$ agreement between the two observers in the classification of the categorical variables of the filtering blebs (Table 1). The intrascleral space dimensions (length and height) and the TDM thickness are shown in Table 2. There was no statistically significant difference $(P>0.05)$ between the quantitative results of the two observers.

There was no significant correlation between the level of IOP at the time of UBM and the length of intrascleral space $\left(r^{2}=0.0016\right)$, the height of the intrascleral space $\left(r^{2}=0.136\right)$, or the thickness of the remaining TDM $\left(r^{2}=0.0009\right)$ (Figures 5-7).

\section{Discussion}

In normal eyes, the main outflow resistance appears to occur at the juxtacanalicular connective tissue layer of the

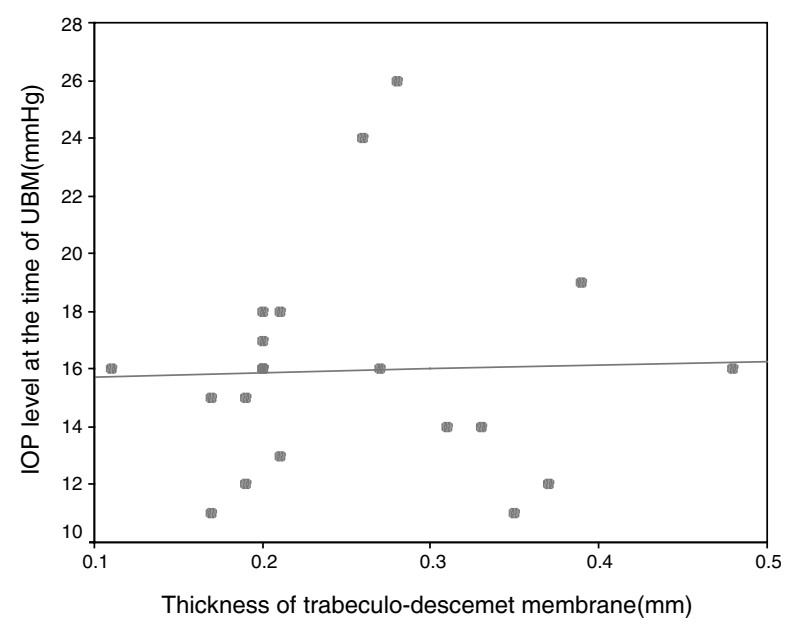

Figure 5 Correlation between IOP at the time of UBM and the minimum thickness of the remaining TDM $\left(r^{2}=0.009\right)$.

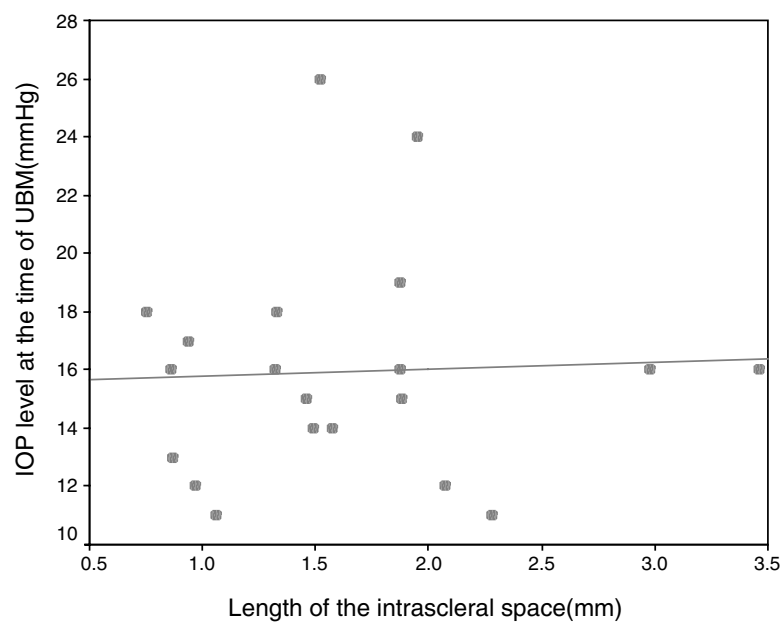

Figure 6 Correlation between IOP at the time of UBM and the length of the remaining TDM $\left(r^{2}=0.001\right)$.

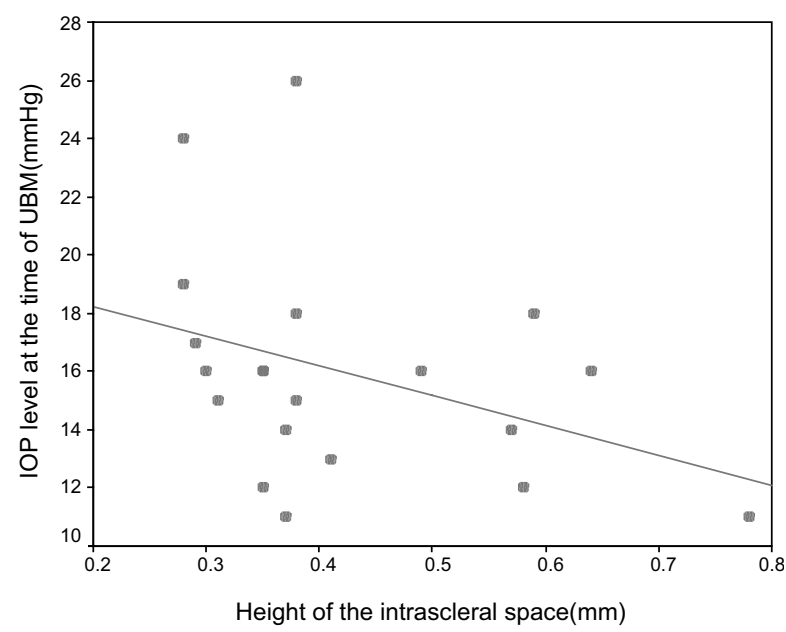

Figure 7 Correlation between IOP at the time of UBM and the height of the remaining TDM $\left(r^{2}=0.13\right)$. 
trabecular meshwork. This area is bypassed by the surgical procedure, allowing the passage of fluid from the anterior chamber to the intrascleral space. ${ }^{16-18}$ Nonpenetrating surgical techniques create a Descemet's window by removing the juxtacanalicular tissue of the Schlemm's canal, leading to lowering the IOP without perforation of the anterior chamber. This in turn may reduce the occurrence of hyphaema, hypotony, choroidal effusion, shallow anterior chamber, intraocular inflammation, and cataract. The reduction of IOP in NPT may be similar or less than the one achieved with standard trabeculectomy. ${ }^{16-22}$

Deep sclerectomy may lead to a reduction of IOP via a number of possible mechanisms, ${ }^{1}$ which might include: creation of a subconjunctival filtering bleb, removal of juxtacanalicular tissue, where it is thought the majority of the flow resistance is located, filtration to the supraciliary/suprachoroidal space, vaulting of residual trabecular meshwork toward the intrascleral cavity leading to widening of the cribriform interspace (similar to laser trabeculoplasty), and development of new aqueous drainage veins in the scleral space, months after deep sclerectomy. ${ }^{23-25}$

UBM can be used to evaluate the morphology and changes of the residual tissue after glaucoma surgery. It provides a subsurface image that cannot be obtained with optical slit-lamp examination. It permits the visualization of the postoperative area and measurements of the thickness of the TDM and the dimensions of the intrascleral space. ${ }^{15}$

Most previous UBM studies evaluated the surgical area in the early postoperative period. The presence of intrascleral space and a thin remaining TDM was a common finding. Negri-Aranguren ${ }^{26}$ in a recent study of UBM after vicocanalostomy found an intrascleral space in all patients $(n=23) 4$ months after surgery and in about $50 \%$ of patients after 13 months. Roters et $a^{27}$ in a similar study found the space in most of the cases 1 month after surgery. Marchini, ${ }^{12}$ in another study on UBM after deep sclerectomy with reticulated hyaluronic acid implants, described an association between the presence of the intrascleral space, the reflectivity of the conjunctival bleb, the supraciliary hypoechoic area, and the IOP lowering outcome.

The volume of the intrascleral space did not appear to be associated with IOP control in previous reports. ${ }^{26,27}$ In this study, the presence of an intrascleral space was a common finding. However, there was no statistically significant relationship between the level of IOP and the dimensions of the intrascleral space. One patient with a successful outcome had no intrascleral space and in another two with failed surgery this space was present.

There are no published data regarding the correlation between the thickness of TDM and the level of IOP. In this study, there was no significant correlation between these variables.

The significance of the suprachoroidal hyporeflective area is unclear. This UBM sign may represent a barely raised detachment of the ciliary body early in the postoperative period. Aqueous humour may leak out via the floor of a sclerectomy thinned by the surgical manoeuvre and cause fluid accumulation in the suprauveal space. Choiu et $a l^{13}$ stated that its presence was associated with lower IOP that was statistically significant at 2 and 3 months postoperatively. Roters et $a l^{27}$ detected the suprachoroidal hyporeflective area in six patients $(n=15) 1$ year after viscocanalostomy, but its presence was not associated with the surgical success. Negri-Aranguren ${ }^{26}$ in a similar study considered that this finding, observed only in two of 23 cases, could not support a major role for the uveal pathway. Marchini et $a l^{12}$ found this area in 18 out of 30 cases 1 year after deep sclerectomy with implant. Kazakova et al reported a significant correlation between suprachoroidal outflow and IOP control in deep sclerectomy with collagen implant. ${ }^{15}$ In the current study, a suprachoroidal hyporeflective area was not found in any case after approximately 1 year of follow-up.

Subconjunctival filtering blebs with variable reflectivity have commonly been found in the short and midterm postoperative periods after nonpenetrating trabeculectomy. The most common type was L-type (Low reflective). ${ }^{15,18,27}$ However, Negri-Aranguren et $a l^{26}$ did not find filtering blebs, except in one case $(n=23), 7-9$ months after viscocanalostomy. Kazakova et al ${ }^{15}$ reported the presence of a low-reflective diffuse subconjunctival space indicating persistent filtration after long-term follow-up after deep sclerectomy with collagen implant.

In this study, filtering blebs were detected in $90.9 \%$ of cases. H-type (high reflective) was the most frequent type $(50 \%)$. The presence and type of filtering bleb were not associated with the surgical outcome. It is interesting to mention that surgery failed in three patients, two had $\mathrm{H}$ type bleb and in the other no filtering bleb was detected. Moreover, surgery was successful in one patient with no filtering bleb detected by UBM. Other studies have also failed to find an association between the filtering bleb and the level of IOP. ${ }^{26,27}$ Many cases with encapsulated and flat blebs had a low IOP. Roters $e t \mathrm{al}^{27}$ suggested that even a large low-reflective filtering bleb, 1 month after viscocanalostomy, was not indicative of good IOP control as two eyes with low-reflective filtering bleb needed additional surgery.

Interobserver variability was evaluated in this study as UBM findings may vary considerably and may be affected by subjective interpretation of visualized landmarks. Tello et $a l^{28}$ stated that individual examiners appear to be internally reliable when obtaining repeated 
measurements from the same scan on multiple occasions, indicating that intraobserver reproducibility was high, but in fact, when several examiners are compared to each other, a difference in measurement appeared. In the current study, there was no significant difference in the measurements or in the evaluation of qualitative or quantitative variables of the examined area between the two masked observers.

In summary, in this study, it appeared that in most patients there was a continuous aqueous filtration through the surgically excised tissue to an area under the scleral flap; then, the aqueous humour reached the subconjunctival space. However, the surgical outcome was not related to the UBM findings of the surgical area. Suprachoroidal flow did not appear to have a role in the long-term reduction of IOP; however, we cannot rule out the possibility of its presence in the early postoperative period. Although UBM provides detailed information regarding the morphology of the anterior segment of the eye, it seems to have limitations when it is used to evaluate mechanisms of aqueous outflow in this deep sclerectomy.

\section{References}

1 Netland PA. Ophthalmic Technology Assessment Committee Glaucoma Panel, American Academy of Ophthalmology. Nonpenetrating glaucoma surgery. Ophthalmology 2001; 108: 416-421.

2 Kranzov MM. Sinusotomy: foundation, results, prospects. Trans Am Ophthalmol Otolaryngol 1972; 76: 369-374.

3 Tan JCH, Hitchings RA. Non-penetrating glaucoma surgery: the state of play. Br J Ophthalmol 2001; 85: 234-237.

4 Zimmermann TJ, Kooner KS, Ford VJ, Olander KW, Mandlekorn RM, Rawlings EF et al. Trabeculectomy vs nonpenetrating trabeculectomy: a retrospective study of two procedures in phakic patients with glaucoma. Ophthalmic Surg 1984; 12: 227-229.

5 Fyodorov SN. Non-penetrating deep sclerectomy in open angle glaucoma. Eye Microsurg 1989; 1: 52-55.

6 Kozlov VI, Bargov SN, Anisimova SY, Opsipov AV, Mogilevtsev W. Non-penetrating deep sclerectomy with collagen. Eye Microsurg 1990; 3: 44-46 (in Russian).

7 Sanchez E, Schnyder CC, Mermoud A. Deep sclerectomy: results with and without collagen implant. Int Ophthalmol 1997; 20: 157-162.

8 Arenas E. Trabeculectomy ab externo. Highlights Ophthalmol 1991; 19: 59-66.

9 Mermoud A, Karlen M-E, Schnyder CC, Sickenberg M, Chiou AG, Hedigner SE et al. Nd: YAG gonipuncture after deep sclerectomy with collagen implants. Ophthalmic Surg Laser 1999; 30: 120-125.

10 Stegmann RC, Pienaar A, Miller D. Viscocanalostomy for open angle glaucoma in black african patients. J Cataract Refract Surg 1999; 25: 316-321.

11 Pavlin C, Harasiewicz K, Sherar M, Foster FS. Clinical use of ultrasound biomicroscopy. Ophthalmology 1991; 98: 287-295.

12 Marchini G, Marraffa M, Brunelli C, Morbio R, Bonomi L. Ultrasound biomicroscopy and intraocular-pressure- lowering mechanisms of deep sclerectomy with reticulated hyaluronic acid implant. J Cataract Refract Surg 2001; 27(4): 507-517.

13 Chiou AGY, Mermoud A, Underdahl JP, Schnyder CC. An ultrasound biomicroscopic study of eyes after deep sclerectomy with collagen implant. Ophthalmology 1998; 105: 746-750.

14 Yamamoto T, Sakuma T, Kitakawa Y. An ultrasound biomicroscopic study of filtering blebs after mitomycin C trabeculectomy. Ophthalmology 1995; 102: 1770-1776.

15 Kazakova D, Roters S, Schnyder CC, Achache F, Jonescucuypers C, Mermoud A et al. Ultrasound biomicroscopy images: long-term results after deep sclerectomy with collagen implant. Graefes Arch Clin Exp Ophthalmol 2002; 240: 918-923.

16 El Sayyad F, Helal M, El-Kholify H, Khalil M, El-Maghraby A. Non-penetrating deep sclerectomy versus trabeculectomy in bilateral primary open angle glaucoma. Ophthalmology 2000; 107: 1671-1674.

17 Chiselita D. Non-penetrating deep sclerectomy versus trabeculectomy in primary open-angle glaucoma surgery. Eye 2001; 15: 197-201.

18 Ambresin A, Shaarawy T, Mermoud A. Deep sclerectomy with collagen implant in one eye compared with trabeculectomy in the other eye of the same patient. J Glaucoma 2002; 11: 214-220.

19 Luke C, Dietlein TS, Jacobi PC et al. A prospective randomized trial of viscocanalostomy versus trabeculectomy in open-angle glaucoma: a 1-year follow-up study. J Glaucoma 2002; 11: 294-299.

20 O'Brart DP, Rowlands E, Islam N, Noury AM. A randomised, prospective study comparing trabeculectomy augmented with antimetabolites with a viscocanalostomy technique for the management of open angle glaucoma uncontrolled by medical therapy. Br J Ophthalmol 2002; 86 : 748-754.

21 Carassa RG, Bettin P, Fiori M, Brancato R. Viscocanalostomy versus trabeculectomy in white adults affected by openangle glaucoma: a 2-year randomized, controlled trial. Ophthalmology 2003; 110: 882-887.

22 Kobayashi H, Kobayashi K, Okinami S. A comparison of the intraocular pressure-lowering effect and safety of viscocanalostomy and trabeculectomy with mitomycin $\mathrm{C}$ in bilateral open-angle glaucoma. Graefes Arch Clin Exp Ophthalmol 2003; 241: 359-366.

23 Bill A. Some aspects of aqueous humour drainage. Eye 1993; 7: 14-19.

24 Vaudaux J, Mermoud A. Aqueous humour dynamics in non-penetrating filtering surgery. Invest Ophthalmol Vis Sci 1997; 38: S1064.

25 Johnson DH, Johnson M. How does non-penetrating glaucoma surgery work? Aqueous outflow resistance and glaucoma surgery. J Glaucoma 2001; 10: 55-67.

26 Negri-Aranguren I, Croxatto O, Grigera DE. Midterm ultrasound biomicroscopy findings in eyes with successful viscocanalostomy. J Cataract Refract Surg 2002; 28(5): 752-757.

27 Roters S, Luke C, Jonescu-Cuypers CP, Engels BF, Jacobi PC, Konen $\mathrm{W}$ et al. Ultrasound biomicroscopy and its value in predicting the long-term outcome of Viscocanalostomy. $\mathrm{Br} \mathrm{J}$ Ophthalmol 2002; 86: 997-1001.

28 Tello C, Liebmann J, Potash SD, Cohen H, Ritch R. Measurement of ultrasound bimicroscopy images; intraobserver and interobservers reliability. Invest Ophthalmol Vis Sci 1994; 35: 3549-3552. 